\title{
Erratum to: The reconstructive challenges of electrical burns to the scalp: a case series
}

\author{
Sarah Strathie Page ${ }^{1}$ - Alexandra Murray ${ }^{1}$. Thomas Jovic ${ }^{1}$. \\ Martin Ball $^{1} \cdot$ Jeremy Rawlins ${ }^{1}$
}

Published online: 14 April 2015

(C) Springer-Verlag Berlin Heidelberg 2015

Erratum to: European Journal of Plastic Surgery

DOI 10.1007/s00238-015-1072-7

The original version of this article contained a mistake in the author name. The author's full name is Sarah Strathie Page with "Strathie Page" being the surname. The author Sarah Strathie Page should be presented as S. Strathie Page in the affiliation section and not S. S. Page.

The online version of the original article can be found at http://dx.doi.org/ 10.1007/s00238-015-1072-7.

\footnotetext{
S. Strathie Page

sarah.strathiepage@health.wa.gov.au

1 Burns Unit, Royal Perth Hospital, Wellington Street,

Perth 6000, Western Australia, Australia
} 